\section{PAEDIATRIC ONE-DAY ADMISSION - IS IT NECESSARY AND WHY?}

Jing Zhan Lock, Jing Zhan Lock, Zi Xean Khoo. Singapore

10.1136/bmjpo-2021-RCPCH.190

Background Paediatric patients being admitted to the inpatient units from the emergency departments (EDs) are increasing across many developed countries. However, despite this increase in the proportion of admissions, the mean inpatient length of stay has fallen significantly.

Objectives This study aimed to assess the necessity of one-day paediatric admissions in Singapore. An unnecessary admission is defined as one with no diagnostic test ordered, intravenous medication administered, therapeutic procedure performed or specialty review made in the inpatient unit. With a better understanding, interventions can be implemented to reduce the number of unnecessary admissions.

Methods We retrospectively reviewed the medical records of paediatric patients who were admitted from a general ED of an adult tertiary hospital to a paediatric tertiary hospital within the same healthcare cluster between 1 August 2018 and 30 April 2020. Paediatric patients were defined as age less than 16 years old. One-day admission was defined as an inpatient stay of less than 24 hours from time of admission to discharge. Data fields including demographic, diagnosis, reason for admission, interventions and treatment procedures performed at both the emergency department and inpatient unit were captured in a standardised data collection form and analysed.

Results During the study period, there were 3944 paediatric attendances at the general ED. One thousand one hundred and sixty one $(8.3 \%)$ paediatric patients were admitted to the inpatient unit of the paediatric tertiary hospital. Among these, $481(41.4 \%)$ were one-day admissions. Upper respiratory tract infection $(62,12.9 \%)$, gastroenteritis $(60,12.5 \%)$ and head injury $(52,10.8 \%)$ were the three most common conditions. The three most common reasons for ED admissions were for inpatient treatment $(203,42.2 \%)$, inpatient monitoring (185, $38.5 \%)$ and inpatient diagnostic investigations (32, 12.3\%). However, 96 (20.0\%) one-day admissions (20.0\%, $n=96)$ were unnecessary with no diagnostic test ordered, intravenous medication administered, therapeutic procedure performed or specialty review made in the inpatient unit.

Conclusions A fifth of paediatric one-day admission in Singapore has been shown to be unnecessary and reasons behind these admissions were multifactorial. A detailed understanding of these reasons can allow us to propose interventions to safely slow down and perhaps reverse this trend.

\section{OUTCOMES OF DIET CONTROL IN GESTATIONAL DIABETES DURING PREGNANCY WITH COUNSELLING AND FOOD NUTRITION MOBILE APPLICATION}

Isabella Liu, Le Ye Lee, Jia Ming Low. Singapore

\subsection{6/bmjpo-2021-RCPCH.191}

Background Poorly controlled Gestational Diabetes Mellitus (GDM) is associated with complications in both mother and infant. The first line of management for these women is dietary modifications but maintaining a healthy and well-balanced diet can be a huge feat to some. Together with the Dietetics
Department, we provided counselling and used a food diary mobile application to help pregnant women with GDM to track their dietary intake so as to improve their diabetic control.

Objectives Our objective is to report the clinical profiles, dietary habits, and outcomes of pregnancy of these recruited women.

Methods A prospective pilot of 30 pregnant women with GDM diagnosed after Oral Glucose Tolerance test at 26 weeks of pregnancy were recruited from April 2019 to June 2020. All received counselling by a diabetic nurse and dietician, and their caloric and nutritious intakes were recorded in the food diary mobile application. Their infants' outcomes, birth weight and blood glucose levels were also tracked. Means were presented with standard deviations for the continuous and normally distributed data.

Results There were 17 Chinese, 10 Malay, and 3 Indian pregnant women with GDM reviewed. The mean maternal weight, height and BMI of these women were $67.4 \mathrm{~kg}$ (SD: 12.5), $1.60 \mathrm{~m}$ (SD: 0.08), $26.1 \mathrm{~kg} / \mathrm{m} 2$ (SD: 4.4) at recruitment. Their mean age was 32.6 years old (SD: 3.8 ). Their median HbA1c was $5.4 \mathrm{~mol} / \mathrm{dL}$. The average daily caloric, protein, fat, carbohydrates, sugar and calcium intake of these women were 1056 kcal (SD: 245.8), $54.5 \mathrm{~g}$ (SD: 15.2), $121 \mathrm{~g}$ (SD: 13.2), $24 \mathrm{~g}$ (SD: 11.8) and $385.4 \mathrm{~g}$ (SD: 215.8) respectively. All of them consumed carbohydrates well below the recommended level which is $175 \mathrm{~g}$ and almost all of their average caloric intakes were below their estimated individualized caloric budget. There was no stillbirth, perinatal asphyxia, serious birth injuries or serious neonatal complications among the infants. The mean birth weight and gestation of the infants were $3.14 \mathrm{~kg}$ (SD: 0.57 ) and 38 completed weeks (SD: 1.5). 2 of them were delivered late premature, and 3 had a birth weight of more than $4 \mathrm{~kg}$. 2 infants had hypoglycemia which responded to supplement feeds without intravenous infusion.

Conclusions These findings suggest that diet-counselling and using a food diary mobile application can encourage pregnant women with GDM to adopt a healthy and well-balanced diet during their pregnancy, which can therefore help to reduce the complications in both mother and infant.

\section{WILL INCREASING THE SCREENING CUT-OFF THYROID STIMULATING HORMONE (TSH) LEVEL IN THE NEWBORN MISS CASES OF CONGENITAL HYPOTHYROIDISM?}

Vanessa Jia Mei Lim, Chin Pin Yeo, Jayme Sau Yeng Wong, Carly Yanlin Wu, Wan Li Ong, Daisy Kwai Lin Chan. Singapore

\subsection{6/bmjpo-2021-RCPCH.192}

Background Newborn screening programs for congenital hypothyroidism $(\mathrm{CH})$ using thyroid stimulating hormone (TSH) as a primary screening test has led to a drastic decrease in untreated $\mathrm{CH}$. There is no optimum screening cut-off level of $\mathrm{TSH}$ to trigger investigations for diagnosis and treatment. In different health systems, the cut-off value could be a standard (fixed) TSH level (such as $10.0 \mathrm{mIU} / \mathrm{L}$ ) or based on the 97.5th, 98.5thor even 99thcentile of a normative TSH distribution. An informal survey of Singapore neonatologists showed that a standard $25.0 \mathrm{mIU} / \mathrm{L}$ is used as the screening cut-off. A pilot study conducted at Singapore General Hospital $(\mathrm{SGH})$ in 2013 showed that umbilical cord TSH values 